\title{
Current Radiological Approach in Thyroid Nodules
}

\section{Duzgun Yildirim1, Deniz Alis², Sabri Sirolu², Cesur Samanci², Fethi Emre Ustabasioglu², Bulent Colakoglu ${ }^{3}$}

\author{
${ }^{1}$ Department of Radiology, Acıbadem Taksim Hospital, Istanbul, Turkey \\ ${ }^{2}$ Department of Radiology, Istanbul University, Cerrahpasa Medical Faculty, Istanbul, Turkey \\ ${ }^{3}$ Department of Radiology, Vehbi Koç Foundation American Hospital, Istanbul, Turkey \\ Email: yildirimduzgun@yahoo.com, drdenizalis@gmail.com, Sabri.sirolu@gmail.com, cesursamanci@gmail.com, \\ ustabasioglu@gmail.com,Blntc@yahoo.com
}

How to cite this paper: Yildirim, D., Alis, D., Sirolu, S., Samanci, C., Ustabasioglu, F.E. and Colakoglu, B. (2017) Current Radiological Approach in Thyroid Nodules. Journal of Cancer Therapy, 8, 423-442. https://doi.org/10.4236/jct.2017.85037

Received: March 3, 2017

Accepted: May 21, 2017

Published: May 24, 2017

Copyright $\odot 2017$ by authors and Scientific Research Publishing Inc. This work is licensed under the Creative Commons Attribution International License (CC BY 4.0).

http://creativecommons.org/licenses/by/4.0/

\begin{abstract}
The aim of this paper is to review the radiological evaluation of thyroid nodules detected with ultrasonography (US), thoroughly and in harmony with the clinical-endocrinological-surgical management. In this review, we tried to summarize the radiological evaluation of nodules detected in the thyroid in compliance with recently published and accepted guidelines, including the most advanced sonographic protocols, emphasizing the indications of crosssectional imaging when needed. In this review important aspects were demonstrated with tables, illustrations and take-home points and technical aspects were emphasized with images and diagrams. We believe that this review, which is concluded with an extensive summary with the support of most recent sources, is an up-to-date and comprehensive evaluation of the broad spectrum of thyroid nodule radiology. We think that the management of any kind of nodule that can be encountered during the US examination can be done successfully by going through the paths described in the pictures, tables and texts in this article.
\end{abstract}

\section{Keywords}

Elastography, Doppler US, Thyroid Cancer, Thyroid Nodule, US

\section{Introduction}

While thyroid carcinoma represents $1 \%$ of all the cancers, $92 \%$ of all the endocrine cancers originate from thyroid. This is important in terms of representing the most pathological group of endocrine organs and in terms of its frequency. Some autopsy series have revealed occult carcinoma or microcarcinoma of the 
thyroid gland in as much as $10 \%$ of the subjects [1].

Routine USG screening for thyroid nodules is not recommended. A thyroid US examination would be appropriate in people with positive findings in physical examination or check-ups (thyroid nodule, painless cervical lymphadenopathy, abnormal thyroid stimulating hormone (TSH) values, etc.) and people with certain risk factors [2]. The radiological and clinical characteristics that increase the risk of malignancy of thyroid nodules are listed in Table 1.

The aim in thyroid imaging is to localize the thyroid gland, and to reveal the anatomy with the size and the contours. After ruling out a parenchymal disease, the presence of a nodule should be assessed. Nodules that are having malignant criteria should be detected and then differential diagnosis regarding the malignancy of the nodules should be assessed according to the clinical-laboratory-radiological properties. Today, nodules are categorized according to their malignancy risk using the thyroid imaging reporting and data system (TIRADS) classification. Nowadays, multi-parametric protocols are being used in nodule US and the decisions for follow-up or further evaluation protocols are based on the malignancy risk obtained from these multi-parametric protocols. Beside thyroid gland examination, radiological evaluation and mapping of cervical lymph nodes is essential during same examination session. Imaging of the complications and residual/recurrent changes in early and late postoperative period and after medical treatment has gained importance. Next generation imaging modalities like molecular tests are about to hit the market and developments in thyroid nodules should be followed closely. In this manner our review has been prepared as an update for the radiological approach to thyroid nodules and a summary guideline for the reader as coverage.

\section{The Sensitivity and the Utilization of the Imaging Modalities for Thyroid Nodules}

Modalities and short explanations are summarized in Table 2. The sensitivities and specificities differ among the modalities. As a beginning the prevalence of nodule detection in thyroid with physical examination is reported to be between $2 \%$ and $6 \%$ in the literature while the prevalence increases to $34 \%-50 \%$ with ultrasonography and to $49 \%-60 \%$ in autopsy series [3].

Scintigraphy is no longer used in clinical practice in differentiating malignant and benign nodules as $5 \%$ of the malignant nodules can appear "hot" and $20 \%$ of the benign nodules can appear "cold" in scintigraphy [4].

Differentiation of diffuse parenchymal diseases as Graves' disease or Hashimoto's thyroiditis can be very easy with scintigraphy. Scintigraphy is also useful in differentiating benign and malign thyroid nodules (Table 3). On the other hand, scintigraphic evaluation of thyroid nodules might prevent radioactive iodine treatment for 2 - 3 months [5] Table 3.

\subsection{Evaluation of Thyroid Nodules with MRI and PET/CT}

Sectional examinations should generally be preferred in cases where sonographic 
Table 1. The radiological and clinical characteristics that increase the risk of malignancy of thyroid nodules are listed in Table 1.

Risk factors for malignancy in patients with thyroid nodules

- History of head and neck RT

- Familial cancer (medullary cancer, MEN2, papillary cancer)

- People younger than 14 or older than 70

- Male gender

- Increase in nodule size

- Hard or fixed nodule, Cervical lymph nodes

- Persistent dysphonia-dysphagia-dyspnea

Table 2. Sensitivity of radiological modalities to detect thyroid nodules.

Sensitivities of modalities for thyroid nodules

(-: not used, + number proportional to the availability of the modality)

- Direct graphs

- Physical examination

- CT

- MRI

- Scintigraphy

- US

- Fine needle biopsy

- Excisional biopsy
- -

- ++

- +

- +++

- ++

- ++++

- +++++

- ++++++

Table 3. Recommendations for scintigraphic evaluation in thyroid diseases.

\section{Situations in which scintigraphy is advised}

- If TSH is low or at the lower limit of normal in presence of nodule(s)

- Iodine deficiency

- To search for any ectopic mediastinal thyroid tissue

- To decide upon the suitability of radioiodine treatment

orientation is impossible such as giant nodular enlargement in the thyroid gland, extension into the mediastinum or local soft tissue invasion. Regardless of an extra criterion, the malignancy potential of a node in the centimetric level seen on tomography was described as $11 \%$. CT can also demonstrate calcifications, irregular contour, invasion and associated lymphadenopathies better than US (Figure 1).

PET/CT is not preferred in the management of thyroid neoplasms, although it can be used as the primary evaluation in cases where the tumor is too undifferentiated to uptake radioiodine. PET/CT is usually involved in thyroid imaging when it demonstrates diffuse or focal uptake in thyroid in examinations done for another cause. While the diffuse involvement of parenchymal is (correlating with TSH and T3-T4 values) interpreted in favor of dysfunction, malignancy risk is up to $40 \%$ in focal uptake (Figure 2). The risk of malignancy increases especially in case of a SUV max value greater than 5.5. Combining the grey-scale, Doppler window and recently elastographic properties in sonography, these nodules are managed successfully with follow-up, biopsy or surgery [6]. 


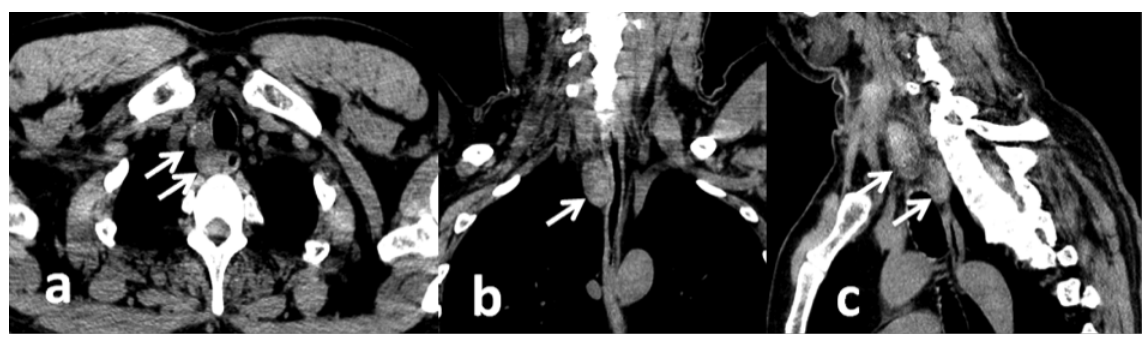

Figure 1. 2 nodular units protruding into the mediastinum and the right tracheoesophageal sulcus in a case with multifocal papillary thyroid cancer. These findings have only been appreciated with multiplanar CT.

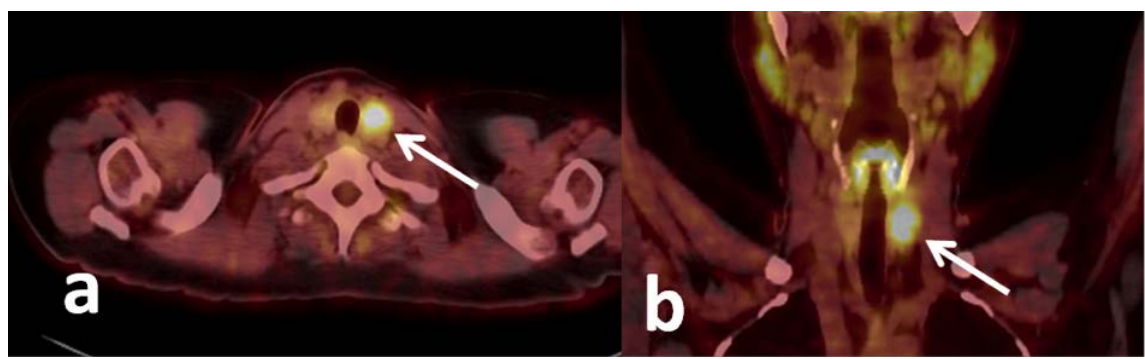

Figure 2. A nodule with a SUV max of 7, 85 in the left lobe of thyroid (papillary thyroid carcinoma).

Mediastinal extension can be evaluated with MRI and CT better than US. MRI has a better soft tissue resolution and it does not expose the patient to X-ray. Non-contrast studies such as diffusion, dynamic contrast study and ASL can also be used when the characterization of the nodules is difficult with US (For example, in diffusion-weighted evaluations, it is likely that nodules with ADC limitation below $0.9 \times 10^{-3} \mathrm{~mm}^{2} / \mathrm{s}$ are malignant, especially papillary type thyroid carcinoma.

\subsection{Ultrasonography}

Ultrasonography has being used as primary imaging modality in the evaluation of thyroid nodules since its first use and its use has increased steadily. Examinations with grey-scale, color Doppler US, power Doppler US, high transducers, strain elastography and eventually shear wave elastography (SWE) have had increasing importance and the addition of guidance for fine needle biopsy has made ultrasonography the top choice in thyroid nodule evaluation. Today, multiparametric ultrasonography protocol with high resolution linear transducer US has increased efficacy with gray scale examination, color Doppler and power Doppler examination, gradually decreasing use of strain elastography and increasingly applied softwares with increasing use of SWE (Figures 3-5) [7]. New studies with CE-US and texture analysis are being done but their effectiveness is debatable.

What is important here is to describe in detail the features of the thyroid gland (volume, parenchyma structure, suspicion of a parenchymal disease, variant lobe extensions in gland contours), detection and assessment of nodules. 


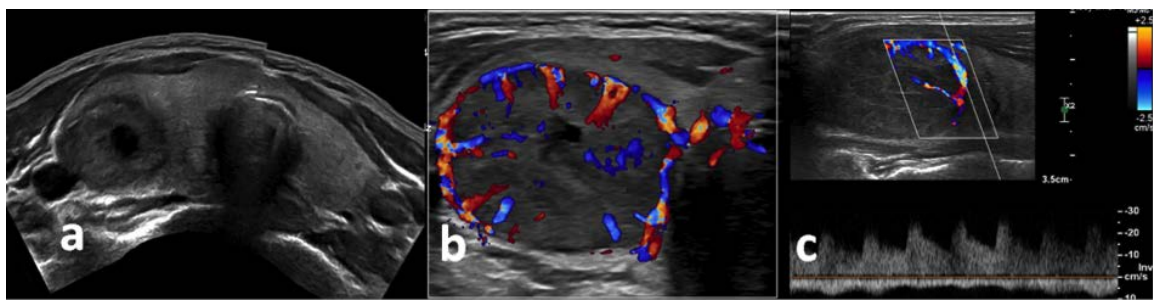

Figure 3. (a) Grey scale (panoramic), (b) Doppler, (c) pulsed Doppler (spectral window) images of a nodule in the right lobe of thyroid gland. The spectral flow of the nodule which has a peripheral vascularization, is anterograde diastolic with a relatively high flow rate.

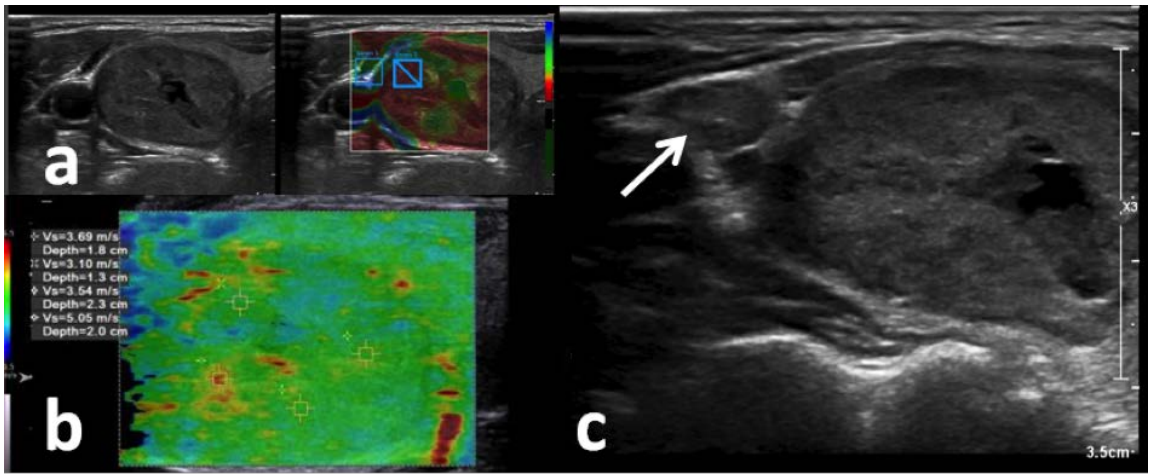

Figure 4. With (a) strain elastography and (b) SWE assessment in the same case, the strain rates and shear wave velocities (SWV) are high for a benign nodule. (c) A metastatic lymph node is also detected in the same level with this nodule at the level 3 in the right cervical chain. Excisional biopsy revealed a follicular variant papillary thyroid cancer.

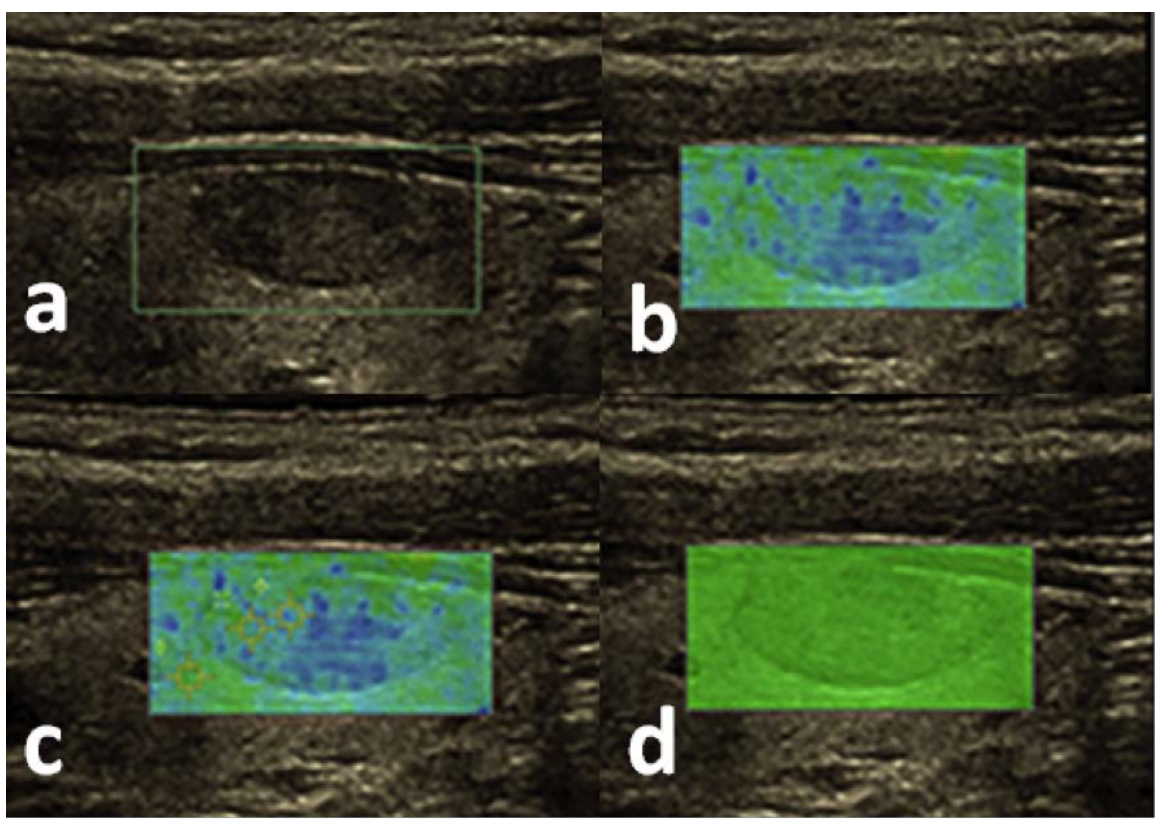

Figure 5. SW maps can be shown with different parameters (velocity, wave travel time, quality map) and checked for a sufficient image quality. (a) ROI window, (b) velocity map, (c) travel time and displacement maps, (d) quality map. 
The characterization of the nodules should be noted with careful and emphatic attention to detailing the dominant nodule properties (gray scale, Doppler, SWE characteristics), FNAB recommendation, and points to be considered during FNAB (nodule placement, probable neural-vascular proximity).

The detailed information regarding the parenchyma, nodule and lymph node status as assessed by the physician doing the sonography should be standardized according to a template and inform the reader clearly. The reason for the examination, technique, equipment, findings, conclusion and suggestions should definitely be included. What should be included in the thyroid nodule assessment form of the radiologist were summarized in Table 4.

If needed, an example template can be used, emphasizing the dominant properties and possible visual information [8].

Pathologies only should not be focused during examinations. Strap muscles in front, laterally carotid sheath containing vagus nerve, carotid artery and jugular vein, recurrent laryngeal nerve, sympathetic plexus nerves, phrenic nerve planes should be included especially in sectional anatomic images [9].

Table 4. What should be included in the thyroid nodule assessment form of the radiologist.

Clinical information-history

- Physical examination findings

- Previous studies

- Risk factors, if any

The modality of the examination

- Device-technical features

- Artifacts and insufficiencies

Findings

- Echotexture, vascularization and volume of the thyroid gland

- Nodule assessment (number, size, localization)

- Risk categorization of the nodule

- Is there any lymphadenopathies especially in stations number II, III, IV or VI

The features to be assessed in the presence of a nodule: Echogenicity (iso-hypo-hyper), halo (thin, thick [ $>2 \mathrm{~mm}$ ], absent, disrupted), calcification (macro-amorphous, micro, eggshell-disrupted or undisrupted) capsular proximity, shape-contour (oval-circular, taller of wider), content (solid or cystic), margins (regular, irregular, microlobulated, spiculated), vascularization (absent, peripheral, central, mixed)

Last control; are there any of the following findings?

- MARKEDLY HyPOECHOIC

- MiCROCALCIFICATION

- IRREGULAR CONTOURS

- eXtrathyroidal eXtension

- taller than wide

- METASTATIC LYMPH NODE

Conclusion and suggestions

- Dominant nodule (2 or 3 dominant nodules at most) localization, size and the dominant property

- Comparison with the previous examinations (differences, progression, new feature)

- TIRADS

- Control US or FNAB suggestions 
It should be noted that nodular thyroidal changes can occur as misleading findings of parenchymal diseases. Pseudonodule formations in chronic autoimmune thyroiditis, subacute granulomatous thyroiditis, silent thyroiditis, Riedel thyroiditis, diffuse hyperplastic goiter and Graves' disease can be misleading (Table 5).

Although the differentiation of the nodular and pseudonodular reflections of diffuse parenchymal diseases is based on clinical and laboratory data, ultrasonography can be helpful, pseudonodules in Hashimoto's thyroiditis for example, are isoelastic with the gland. Nodules in patients with thyroiditis, especially subacute granulomatous thyroiditis, can have spiculated contours, hypo-avascular nature and do not exceed the capsule. The fact that these nodules regress in follow-up exams 1 - 3 months later, shows that immediate use of the biopsy alternative may sometimes be incorrect [10]. General classification of thyroid nodules with cytohistopathological correlation was demonstrated in Table 5.

When the features of a thyroid nodule does not resemble the characteristic and conventional-experienced textures of a benign or a malignant tumor; diseases like tuberculosis, sarcoidosis, amyloidosis, fibrosis or metastasis, as well as benign entities like ectopic thymus and ectopic parathyroid should be kept in mind.

Sometimes cystic thyroid nodules can spontaneously regress or take the collapsed or humidified appearance after aspiration, irrigation, RF ablation or spontaneous regression of hematoma and mimic malignant nodules in greyscale images. Elastography can be problem solving in these cases. Characteristically, with SWE and virtual touch imaging (VTI) mapping, these nodules are softer than expected in comparison to the surrounding tissues [11].

Etiopathogenesis of thyroid nodules is not within the scope of this review, however, they occur as the overgrowth of the monoclonal cells. These cells can first have the adenoma stage and then transform to carcinoma and eventually to the undifferentiated and anaplastic forms, or they can progress directly to the anaplastic stages skipping the other steps [12].

Generally, hyperplastic adenomatous or colloid nodules have smooth margins, thin peripheric rim and are iso-hyperechoic. They don't display central vascularization and sometimes moderate peripheral vascularization can be noted (Figure 6).

Nodules having spongiform or honeycomb pattern, that are completely cystic or mostly cystic and the remaining being isohyperechogenic, displaying giraffe pattern, white knight pattern, multiple isohyperechoic nodules and cystic nodules containing small bright echoes are considered almost 100\% benign. Al-

Table 5. General classification of thyroid nodules with cytohistopathological correlation.

- Benign hyperplastic colloid nodules

- Adenomas (follicular more common, papillary less common)

- Malignant thyroid nodules (papillary more common, follicular less common; rarely

undifferentiated and anaplastic types)

- Other tumors (lymphoma, sarcoma, metastasis) 


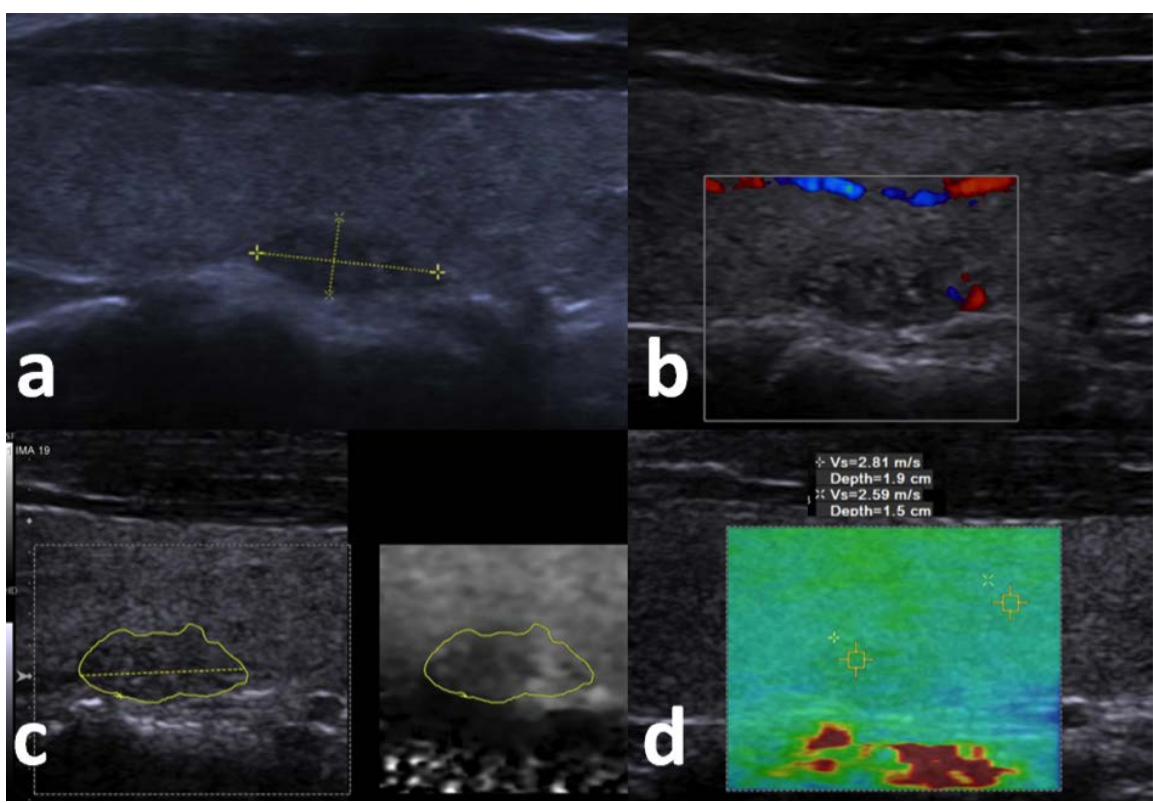

Figure 6. Note that the benign colloid nodule has smooth margins, thin peripheral halo, (b) does not display vascularization in Doppler and isoelastic with the normal parenchyma in (c) VTI and (d) SWV.

though follicular variant of papillary thyroid carcinoma (FVPTC) is similar to adenomas, disruption of the capsule, central vascularization, nodule in nodule appearance and asymmetry in the halo are valuable findings. Note that the histopathologically benign nodules like adenomas can be iso-hyperechogenic, with a peripherally increased vascularization, and a halo without any sign of disruption and asymmetry. Unlike follicular adenomas, follicular carcinomas are more hypoechogenic and are characterized with disruption in the halo contour, asymmetry, central vascularization (sometimes without increase in peripheral vascularization) or only a thick halo of $2-3 \mathrm{~mm}$.

In spite of being separate entities, follicular and papillary cancer groups have subtypes (subgroups, variants) between them that are distinguishable by both immunohistochemical and ,recently, molecular analyses. Among the variability exceeding the aim of this review, some important aspects should be noted. For example Hurthle cell variants have an unfavorable prognosis and tumors with insular type growth pattern have quite fast progression [13].

While the term "differentiated" includes all the follicular and papillary types, the term "anaplastic" is used with further loss of differentiation. Medullary thyroid carcinoma (MTC) is itself a different cancer group and originates from the parafollicular cells of the thyroid and not from the follicular cells. Although medullary thyroid carcinoma has a similar appearance to papillary carcinoma (PTC), it has some distinguishing features. The features can help distinguish PTC-MTC summarized in Table 6 [14].

Anaplastic and undifferentiated carcinomas of thyroid are fast growing heterogenous hypoechoic masses like lymphoma. A fast growing mass in the setting of chronic thyroiditis and linear hyperechogenic septa similar to the appearance 
in lymph node involvement, can raise the suspicion of lymphoma [15]. Thyroid nodules are subjected to risk categorization upon detection using valid guidelines. In this review this categorization is done in accordance with the ATA 2015, AACE/ACE/AME 2016 and TIRADS guidelines [9] [13] [16] [17] [18] [19] [20].

Risk groups in ATA 2016 have less number of groups and have a clearer classification than ATA 2015, because the risk groups in ATA 2015 had relative complexities inhibiting standardization among examiners. All thyroid nodules can be classified into three major groups according to the latest studies (Table 7). The management of the nodules should be determined according to this risk categorization. TIRADS category should be defined combining the clinical, laboratory and radiological findings (Figure 7) [9] [13] [19] [20].

Table 6. MTC has similar features to PTC. The distinguishing features are.

- Macrocalcifications rather than microcalcifications

- Tendency to locate in superolateral portions of upper-middle parts

- More vascularity than PTC

- Larger at the time of diagnosis $(2.3 \pm 1.5 \mathrm{~cm}$ vs. $1.1 \pm 1.1 \mathrm{~cm})$

- Focal cystic degeneration more often than PTC

- Solid component more homogenous than PTC

- Regular margins rather than spiculated contours is possible

- Metastatic lymph nodes of MTC do not display hyperechogenicity

- The strongest distinguisher is serum calcitonin levels.

Table 7. Risk categories that are given in complex form in the latest classifications, and that are gathered under essentially three main groups.

\begin{tabular}{cl}
\hline Risk group & \multicolumn{1}{c}{ Classified properties as a result of } \\
& the cumulative information of the 2015 and 2016 guidelines \\
& - Taller than wide \\
& - Microcalcifications \\
& - Marked hypoechogenicity \\
High risk & - Disruption of the peripheral halo more than the 50\% of the circumfe- \\
thyroid & rence or a thick irregular halo \\
nodule & calcified rim. \\
& - Central vascularization in a solid iso-hypoechoic nodule with a pre- \\
& served halo \\
& - Extrathyroidal extension. \\
& - Associated cervical lymphadenopathy \\
& - Iso-hyperechoic nodule with a peripheral vascularization and pre- \\
Intermediate & served halo. \\
risk thyroid & - Peripheral well preserved egg-shell type rim calcification \\
nodule & in central vascularity \\
& - Intranodular macrocalcification, indetermined hyperechogenic spots, \\
& complete egg-shell calcification \\
& - Increased stiffness
\end{tabular}




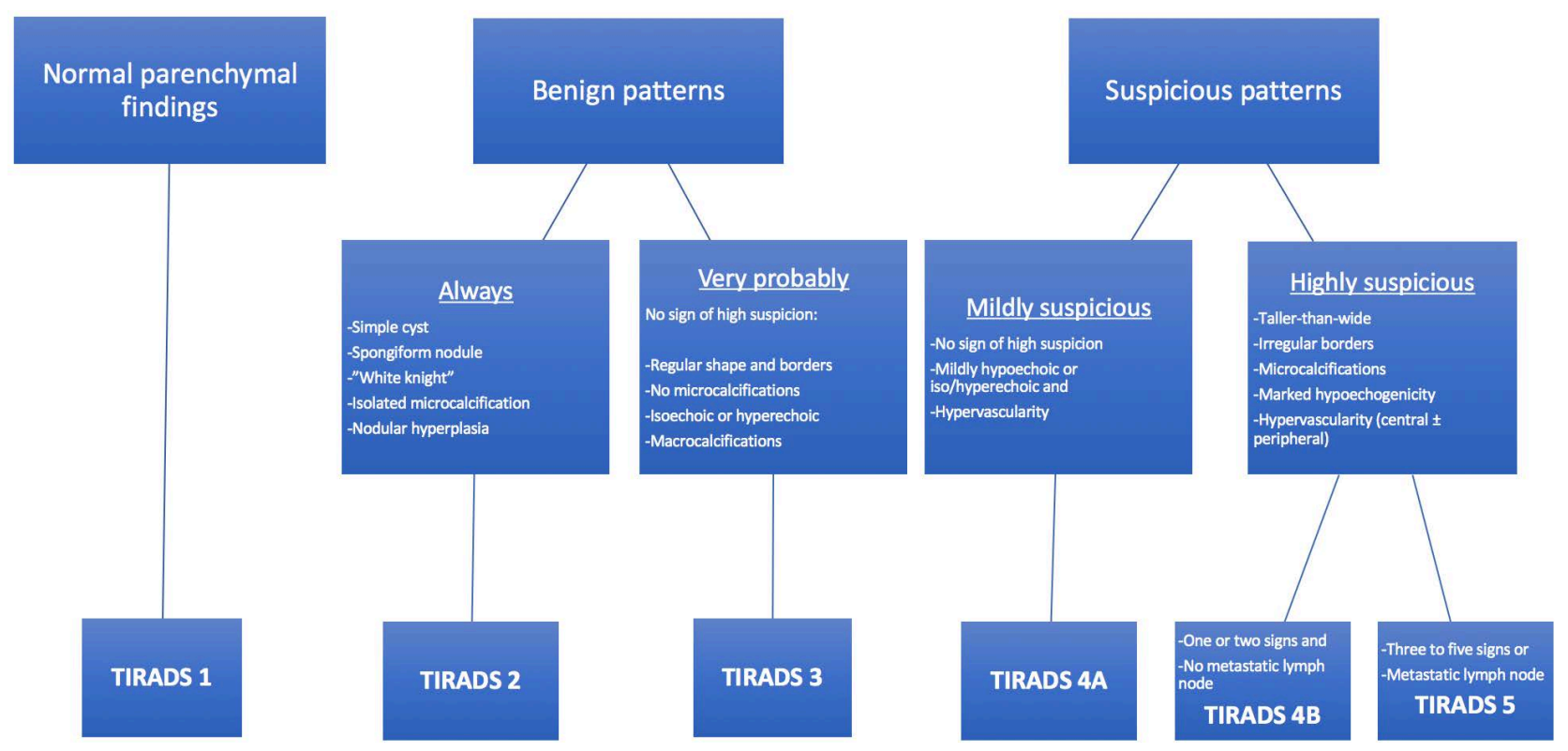

Figure 7. TIRADS categorization of thyroid nodules.

The risk of malignancy reaches $90 \%$ in the presence of these factors, whereas the risk is less than $1 \%$ for a simple thyroid cyst. As these change the management, we should categorize the risks as much as we can unless there appear studies and guidelines against it.

Elastographic measurements have been included in the latest guidelines with increased stiffness, especially the velocity values exceeding $3.1 \mathrm{~m} / \mathrm{s}$, have been categorized TIRADS $4 \mathrm{~b}$ although different cut-off values have been defined in the literature [13] [20].

SWE will continue being the problem solver not only with the risk categorization using the shear wave velocity (SWV) values, but with many other aspects, i.e. comparing the SWE color coded maps of the normal parenchyma and the lesions $[21]$

Sometimes the stiffness of the nodule can be graded using the VTI maps and the risk of malignancy and a possible lymph node metastasis can be predicted [22]. SWE can sometimes present more useful and problem-solving data in the categorization of the thyroid nodules with nondiagnostic grey-scale US findings.

As one of the most promising components of SWE, more should be told about VTI. VTI maps are acquired during the SWE examinations using Acoustic Radiation Force Impulse (ARFI). ARFI is currently the newest imaging mode for detection of mechanical properties of tissue without external compression. ARFI technology includes virtual touch tissue imaging (VTI) and virtual touch tissue and image quantification (VTQ and VTIQ). Micro-displacement is generated in a tissue within the region of interest (ROI) when the commercially available ultrasound transducer launches short-duration (<100 s), high-intensity acoustic pulses to the tissue. The displacement of the tissue depends on its elasticity. The more elastic the tissue is, the more displacement it experiences [1]. The displacement of the tissue within the ROI induced by ARFI is detected and pre- 
sented as a grey-scale image which is called virtual touch tissue imaging (VTI). The harder the tissue is, the darker the image is. The textures of the nodules are graded by using VTI (Table 8). Even the color coded SWV maps and velocity values can be misleading, but VTI can be used more effectively in the future, especially in papillary and follicular microcarcinomas (Figure 8). VTI seems promising as a life saver for the radiologist in the solution of the indeterminate hyperechogenic spots developing in some nodules. In these situations bright foci on VTI which spill over the grey scale projection can be considered benign [23].

This review has several limitations. Referencing main guidelines only, not emphasizing real-time tissue elastography (RTE) and the lack of SWV values in $\mathrm{kPa}$ are the most important of those. The reason for referencing the main guidelines with international validity is to simplify the classifications, to take recent versions into account and not to confuse the reader by mentioning all guidelines. SWE is emphasized in the review instead of RTE because it is more quantitative and operator independent and we wouldn't intend to lead the reader about a method that we don't routinely use in our unit. However there are plenty of valuable studies on RTE and willing readers can research the literature [24] [25] [26] [27].

Several factors that should be keep in mind during SWE examinations were summarized in Table 9.

Table 8. VTI grading in thyroid nodules
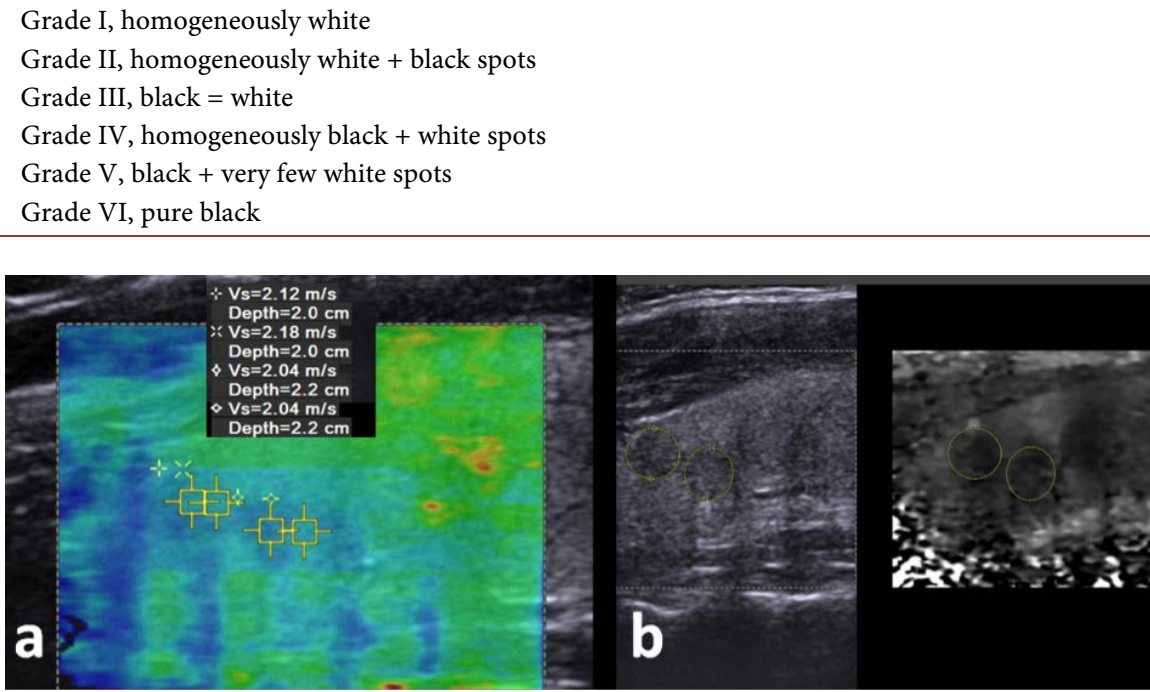

Figure 8. (a) Multiple soft nodules on SWV maps with (b) velocity values in benign limits, displaying high VTI grade.

Table 9. Points to be considered when evaluating thyroid nodules with elastography.

While using elastography;

- Region with motion artifact

- Lesion more superficial than $0.5 \mathrm{~cm}$ or deeper than $4 \mathrm{~cm}$

- Area larger than $8 \mathrm{~cm}$

- Area containing calcification or cystic degeneration. Should be omitted. 
On the other hand we didn't mention CE-US at all because it is used more in the follow-up of RF ablated areas and not preferred for the evaluation of thyroid nodules. Thus it is not mentioned in this review [28] [29].

Actually because the speed of the shear waves in the tissue is measured and systems measuring in $\mathrm{m} / \mathrm{s}$ are being used in our facilities since 2012 , this unit is preferred intentionally. However $\mathrm{m} / \mathrm{s}$ versus $\mathrm{kPa}$ measurements are convertible to each other and suitable for use in both ways [30].

Postoperative management and imaging, FNAB and molecular tests are not emphasized, as they are not in the scope of this review. However a little note should be made here; to avoid complications, parenchymal residues near superior laryngeal artery and nerve are sometimes left behind intentionally. The residual tissues, recurrent hyperplasia, suture granulomas and fibrogranular calcifications should not be confused with malignancy. There are up to date and informative resources on FNAB and postoperative thyroid imaging which the reader can benefit from [3] [31].

Some practical aspects should be paid attention while performing FNAB. First, one should know that FNAB is not the gold standard and it should be noted that in experienced hands,

- $10 \%$ of the biopsy specimens are insufficient.

- Near 2\% false negative (this ratio increases as the size gets close to $4 \mathrm{~cm}$ ) results are obtained. One of the aims of SWE and VTI-ARFI is to prevent missing these lesions.

- Some studies report discordance between FNAB results and surgical specimens in up to $15 \%$ of the cases.

In this case, the best alternative other than repeated FNAB is molecular tests, which is projected to be used routinely in the future [12] [32] [33] [34].

Lastly one of the most important limitations of the review is the fact that cervical lymph node evaluation has not been fully elucidated not to deflect from the main topic. However cervical lymph nodes should be evaluated with accepted size and morphological criteria, especially in level 2a, 3, 4, 5b and 6 stations and in patients with malignant FNAB results. Sampling of these lymph nodes pre or intraoperatively using FNAB and cytology or specimen TG wash-out, the diagnosis of metastasis can be made [2] [35] [36].

FNAB findings are evaluated and interpreted by cytopathologists using a standardized and widely used system (Bethesda classification system.) In this system lesions are classified as nondiagnostic, benign, indeterminate (atypia or follicular lesion of undetermined significance), follicular neoplasm/SFN, suspicious for malignancy and malignant. The next step of the management should be planned according to the FNAB result (Figure 9) [37] [38]. Although it is not in the scope of this review, a few words need to be said upon the molecular tests, since our colored algorithm mentions them. More detailed subgroups or risk categorization can be done using molecular tests on FNAB specimens. With these tests BRAF and RAS mutations are detected in $40 \%$ of PTC cases and PAX8PPARG translocation in $60 \%$ of FTC cases. The frequency of RET mutation in 
familial cases of MTC may reach $95 \%$. TP53 is seen in half of anaplastic type thyroid cancer cases [33].

These tests are different from the immunocytochemical tests which are used when the cytopathological investigations are not diagnostic especially when tumors of nonfollicular cell origin are considered (medullary thyroid carcinoma, lymphoma, metastasis or pathology of parathyroidal origin). Molecular testing studies have especially been commenced in follicular lesions (5\% - 6\% false negativity in FNAB in this indeterminate group warranted evaluation with another test) and these molecular tests will not intend to skip the cytopathological findings, but rather assist them as elastography assists ultrasound.

Herein, reaching the ends of the review, another point to be emphasized at this point is that the approach to papillary microcarcinomas has also been updated. Last guidelines have had improvements on the subject. FNAB is not recommended in nodules smaller than $5 \mathrm{~mm}$ anyway. However FNAB is not recommended in nodules between 5 and $10 \mathrm{~mm}$ if they are not TIRADS $4 \mathrm{~b}$ or 5 , located supcapsularly, located superolaterally with elevated calcitonin levels, the case younger than 40 years, there is no evidence of multifocality or associated lypmhadenopathy, they do not exhibit pathological FDG uptake, and if the SWE velocity values and VTI grades are not pathological. The interesting points in the latest guidelines and literature is that the cases with papillary thyroid microcarcinoma which don't have these properties, which don't have a risky localization (subcapsular, paratracheal) and which don't have associated lymphadenopathy

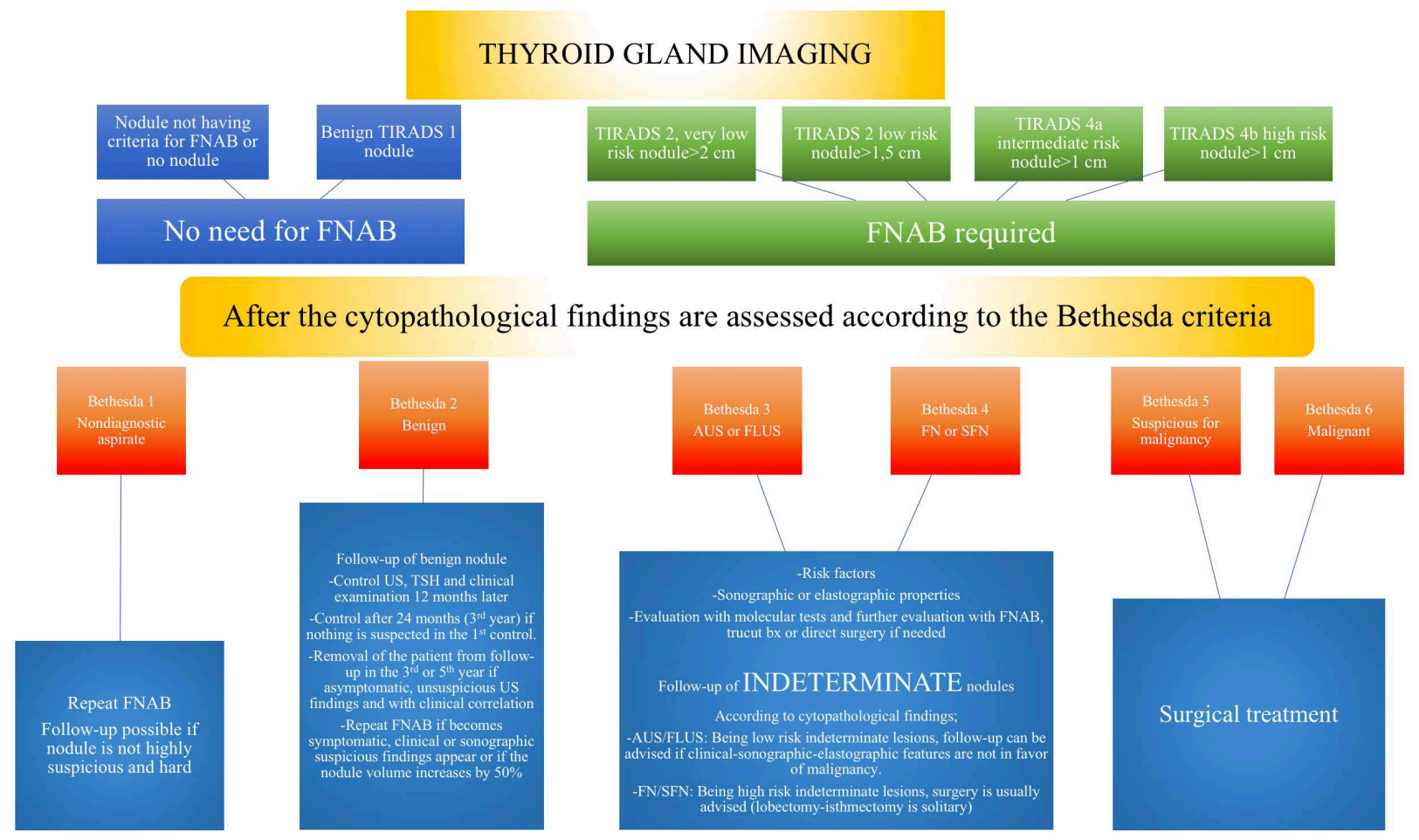

Figure 9. Knowing this table that summarizes the management of the FNAB results after all the risk classifications and TIRADS categorization, would prevent both adverse health consequences for the patient and the medicolegal problems the doctor-facility might face. 
should be kept in a process active surveillance (US control in 6 - 12 month periods) [39] [40]. So, risky nodules should be differentiated considering the parameters determined by large radiological and endocrinological societies that we mentioned and not getting confused by the deceptive radiological findings.

A little word is necessary on the texture analysis which is another of the blessings of technology and which has started to penetrate the radiological examinations. Texture analyses with various programs are beginning to produce standardized-automated and repeatable information about the nodule-parenchymal background structure. However these analyses have not been able to get in to the routine practice and recent guidelines and they need comprehensive trials to prove their effectiveness [41] [42]. Little but significant differences exist on the management of thyroid nodules in pregnancy and children, although they have not been subject to complex algorithms.

Thyroid nodules in pregnancy [13] [43]:

- Diagnostic criteria are the same as nonpregnant adults.

- Medical and radioiodine treatments cannot be used during pregnancy.

- Rebiopsies of the indeterminate cases can be planned during pregnancy.

- The treatment of nonaggressive malignant nodules with no lymph node involvement can be postponed until the birth.

- Thyroid nodules in children [44] [45]:

- Evaluation is the same as adults.

- Cold and hot nodules can both be malignant in this age group.

- Unless purely cystic, $25 \%$ of the nodules are malignant even if they are hot.

\section{Summary of the Evaluation of Thyroid Nodules}

Firstly thyroid nodule evaluations need to be done considering ATA 2015 and its partially updated versions AACE/ACA and AACE/AME May 2016. These evidence based guidelines are intended to aid in the personalized management of patients as well as multidisciplinary cooperation.

Some common questions should be asked the patients in the history taking such as, the age and gender of the patient, history of thyroidal disease for the patient and his/her family, any palpable mass or pain in the neck, presence of dysphonia, dysphagia or dyspnea, symptoms of hyper or hypothyroidism and usage of thyroid supplementation. With sufficient history, sonographic examination begins. First things to examine are the localization of the thyroid gland, its size, structure, vascularization, presence of a nodule or a cervical lymphadenomegaly.Nodules detected in sonography are then categorized according to their properties like localization, position, diameter, shape, contours, internal structure and echotexture, vascularity and elasticity. Elastography should be used together with grey scale findings and not alone (guidelines have not made any distinction between strain and shear wave elastography). Suspicious lymph nodes in the neck are reported with their number, level, shape, contour and echogenicity.CE-US can be used in the ablated zone after RF ablation but it is not used in the evaluation of thyroid nodules. 
Cytology is reported in line with the Bethesda classification as nondiagnostic, benign, indeterminate (atypia or follicular lesion of undetermined significance), follicular neoplasm/SFN, suspicious for malignancy or malignant. In order to decrease the false negativity, at least 2 risky regions in the nodule should be targeted, especially vascularized, stiff solid components on ultrasound examination. In case of insufficient sampling, high risk nodules should be reevaluated with FNAB and if the result of the second FNAB is reported as Bethesda 3, decisions of close follow-up, tru-cut or excision can be made.

In case of non-diagnostic cytopathological results, especially when there is a suspicion of a tumor of nonfollicular origin (medullary thyroid carcinoma, parathyroidal pathology, lymphoma or metastasis), immunocytochemical investigations can be made. Follicular lesions have a 5\% - 6\% false negativity and evaluation with another test in this indeterminate group is needed. Thus there began a tendency for evaluation with molecular tests. These tests will not replace cytopathology, rather aid it as elastography does ultrasound. Molecular tests seem promising as an aid to FNAB.

Summary of the FNAB indications and what to do next;

- Nodules smaller than $5 \mathrm{~mm}$ should be followed-up, regardless of their sonographic findings.

- Nodules that are 5 to $10 \mathrm{~mm}$ in size with suspicious findings can be followed-up closely (watchful waiting, active surveillance) or biopsied. FNAB is performed if the nodule is located subcapsularly or paratracheally, if there is a suspicion for lymph node involvement or extrathyroidal extension, a personal or family history of thyroid cancer, or if there are suspicious clinical findings like dysphonia.

- FNAB (before surgical treatment) is recommended to high risk nodules that are larger than $10 \mathrm{~mm}$, intermediate risk nodules that are larger than $20 \mathrm{~mm}$, or low risk nodules that are larger than $20 \mathrm{~mm}$ and have a tendency to grow.

Scintigraphy is recommended if TSH is subnormal. Functional nodules in scintigraphy are not targeted in FNAB.

- In multinodular lesions usually 2 (maximum 3) nodules should be targeted for FNAB. More than 2 nodule biopsied is not preferred. Hypo or isoactive dominant nodules in scintigraphy are targeted.

- Tg wash-out is studied sampling the lymph node together with the nodule in case of suspicious lymph nodes, especially in the central and lateral compartments. As a rule, stiff and vascularized solid components of the nodules are targeted.

- CT and MRI demonstrate local invasion, mediastinal extension and distant metastases. All nodules uptaking FDG in PET/CT should be further evaluated with biopsy.

Summarizing all the content of this review in conjunction with current references, an objective TIRADS categorization of the nodule as shown in Table 9 and an approach such as Figure 9 will provide algorithm-dependent evaluation in the presence of all the problems encountered. 


\section{Conclusion}

In order to increase the quality of the thyroid nodule examination and help the patients and clinicians as much as possible, $18 \mathrm{mHz}$ transducers should be used in grey scale imaging; Doppler findings should be included; SWE and VTI should be used instead of RTE; extrathyroidal spread and cervical lymphadenopathies should always be assessed and in certain cases CT and MRI should be benefited from. One last word would be about updating oneself without abandoning the routine practice and experiences as Alvin Toffler said in 1990, "The illiterate of the 21st century will not be those who cannot read and write, but those who cannot learn, unlearn, and relearn".

\section{References}

[1] Paschou, S.A., Vryonidou, A. and Goulis, D.G. (2017) Thyroid Nodules: A Guide to Assessment, Treatment and Follow-Up. Maturitas, 96, 1-9.

https://doi.org/10.1016/j.maturitas.2016.11.002

[2] Mitchell, A.L., Gandhi, A., Scott-Coombes, D. and Perros, P. (2016) Management of Thyroid Cancer: United Kingdom National Multidisciplinary Guidelines. The Journal of Laryngology \& Otology, 130, S150-S160. https://doi.org/10.1017/s0022215116000578

[3] Franco Uliaque, C., Pardo Berdún, F.J., Laborda Herrero, R. and Lórenz, C.P. (2016) Usefulness of Ultrasonography Is the Evaluation of Thyroid Nodules. Radiologia, 58, 380-388. https://doi.org/10.1016/j.rx.2016.06.003

[4] Wiyanto, J., Kartamihardja, A.H. and Nugrahadi, T. (2016) Can Ultrasound Predict Malignancy in Patient with Thyroid Cold Nodule? World Journal of Nuclear Medicine, 15, 179-183.

[5] Yürekli, Y., Cengiz, A. and Güney, E. (2015) Graves Disease Induced by Radioiodine Therapy for Toxic Nodular Goiter: A Case Report. Molecular Imaging and Radionuclide Therapy, 24, 135-137. https://doi.org/10.4274/mirt.74046

[6] Şencan Eren, M., Özdoğan, Ö., Gedik, A., Ceylan, M., Güray Durak, M., Seçil, M., Koçdor, M.A., Çömlekçi, A. and Durak, H. (2016) The Incidence of 18F-FDG PET/CT Thyroid Incidentalomas and the Prevalence of Malignancy: A Prospective Study. Turkish Journal of Medical Sciences, 46, 840-847.

https://doi.org/10.3906/sag-1503-26

[7] Baser, H., Cakir, B., Topaloglu, O., Alkan, A., Polat, S.B., Dogan, H.T., Yazicioğlu, M.O., Aydin, C. and Ersoy, R. (2017) Diagnostic Accuracy of Thyroid Imaging Reporting and Data System in the Prediction of Malignancy in Nodules with Atypia and Follicular Lesion of Undetermined Significance Cytologies. Clinical Endocrinology, 86, 584-590.

[8] Sturgeon, C., Yang, A. and Elaraj, D. (2016) Surgical Management of Lymph Node Compartments in Papillary Thyroid Cancer. Surgical Oncology Clinics of North America, 25, 17-40. https://doi.org/10.1016/j.soc.2015.08.013

[9] Shin, J.H., Baek, J.H., Chung, J., Ha, E.J., Kim, J.H., Lee, Y.H., Lim, H.K., Moon, W.J., Na, D.G., Park, J.S., Choi, Y.J., Hahn, S.Y., Jeon, S.J., Jung, S.L., Kim, D.W., Kim, E.K., Kwak, J.Y., Lee, C.Y., Lee, H.J., Lee, J.H., Lee, J.H., Lee, K.H., Park, S.W. and Sung, J.Y., Korean Society of Thyroid Radiology (KSThR) and Korean Society of Radiology (2016) Ultrasonography Diagnosis and Imaging-Based Management of Thyroid Nodules: Revised Korean Society of Thyroid Radiology Consensus Statement and Recommendations. Korean Journal of Radiology, 17, 370-395. 
https://doi.org/10.3348/kjr.2016.17.3.370

[10] Pan, F.S., Wang, W., Wang, Y., Xu, M., Liang, J.Y., Zheng, Y.L., Xie, X.Y. and Li, X.X. (2015) Sonographic Features of Thyroid Nodules That May Help Distinguish Clinically Atypical Subacute Thyroiditis from Thyroid Malignancy. Journal of Ultrasound in Medicine, 34, 689-696. https://doi.org/10.7863/ultra.34.4.689

[11] Lacout, A., Chevenet, C. and Marcy, P.Y. (2016) Mummified Thyroid Syndrome. AJR. American Journal of Roentgenology, 206, 837-845. https://doi.org/10.2214/AJR.15.15267

[12] Wylie, D., Beaudenon-Huibregtse, S., Haynes, B.C., Giordano, T.J. and Labourier, E. (2016) Molecular Classification of Thyroid Lesions by Combined Testing for miRNA Gene Expression and Somatic Gene Alterdations. The Journal of Pathology: Clinical Research, 2, 93-103. https://doi.org/10.1002/cjp2.38

[13] Gharib, H., Papini, E., Garber, J.R., Duick, D.S., Harrell, R.M., Hegedüs, L., Paschke, R., Valcavi, R. and Vitti, P. (2016) American Association of Clinical Endocrinologists, American College of Endocrinology, and Associazone Medici Endocrinologi Medical Guidelines for Clinical Practice for the Diagnosis and Management of Thyroid Nodules-2016 UPDATE. Endocrine Practice, 22, 1-60.

[14] Corsello, S.M., Lovicu, R.M., Migneco, M.G., Rufini, V. and Summaria, V. (2000) Diagnostic Approach, Genetic Screening and Prognostic Factors of Medullary Thyroid Carcinoma. Rays, 25, 257-266.

[15] Salama, J.K., Golden, D.W., Yom, S.S., Garg, M.K., Lawson, J., McDonald, M.W., Quon, H., Ridge, J.A., Saba, N., Smith, R.V., Worden, F., Yeung, A.R. and Beitler, J.J. (2014) ACR Appropriateness Criteria ${ }^{\circledR}$ Thyroid Carcinoma. Oral Oncology, 50, 577-586. https://doi.org/10.1016/j.oraloncology.2013.12.004

[16] Haugen, B.R., Alexander, E.K., Bible, K.C., Doherty, G.M., Mandel, S.J., Nikiforov, Y.E., Pacini, F., Randolph, G.W., Sawka, A.M., Schlumberger, M., Schuff, K.G., Sherman, S.I., Sosa, J.A., Steward, D.L., Tuttle, R.M. and Wartofsky, L. (2016) 2015 American Thyroid Association Management Guidelines for Adult Patients with Thyroid Nodules and Differentiated Thyroid Cancer: The American Thyroid Association Guidelines Task Force on Thyroid Nodules and Differentiated Thyroid Cancer. Thyroid, 26, 1-133. https://doi.org/10.1089/thy.2015.0020

[17] Horvath, E., Silva, C.F., Majlis, S., Rodriguez, I., Skoknic, V., Castro, A., Rojas, H., Niedmann, J.P., Madrid, A., Capdeville, F., Whittle, C., Rossi, R., Domínguez, M. and Tala, H. (2016) Prospective Validation of the Ultrasound Based TIRADS (Thyroid Imaging Reporting And Data System) Classification: Results in Surgically Resected Thyroid Nodules. European Radiology, 1-10.

https://doi.org/10.1007/s00330-016-4605-y

[18] Srinivas, M.N., Amogh, V.N., Gautam, M.S., Prathyusha, I.S., Vikram, N.R., Retnam, M.K., Balakrishna, B.V. and Kudva, N. (2016) A Prospective Study to Evaluate the Reliability of Thyroid Imaging Reporting and Data System in Differentiation between Benign and Malignant Thyroid Lesions. Journal of Clinical Imaging Science, 6, 5. https://doi.org/10.4103/2156-7514.177551

[19] Yi, K.H. (2016) The Revised 2016 Korean Thyroid Association Guidelines for Thyroid Nodules and Cancers: Differences from the 2015 American Thyroid Association Guidelines. Endocrinology and Metabolism (SeouI), 31, 373-378. https://doi.org/10.3803/EnM.2016.31.3.373

[20] Russ, G., Bigorgne, C., Royer, B., Rouxel, A. and Bienvenu-Perrard, M. (2011) The Thyroid Imaging Reporting and Data System (TIRADS) for Ultrasound of the Thyroid. Journal de Radiologie, 92, 701-713. https://doi.org/10.1016/j.jradio.2011.03.022 
[21] Yildirim, D., Gurses, B., Gurpinar, B., Ekci, B., Colakoglu, B. and Kaur, A. (2011) Nodule or Pseudonodule? Differentiation in Hashimoto's Thyroiditis with Sonoelastography. Journal of International Medical Research, 39, 2360-2369. https://doi.org/10.1177/147323001103900636

[22] Xu, J.M., Xu, X.H., Xu, H.X., Zhang, Y.F., Guo, L.H., Liu, L.N., Liu, C., Bo, X.W., Qu, S., Xing, M. and Li, X.L. (2016) Prediction of Cervical Lymph Node Metastasis in Patients with Papillary Thyroid Cancer Using Combined Conventional Ultrasound, Strain Elastography, and Acoustic Radiation Force Impulse (ARFI) Elastography. European Radiology, 26, 2611-2622. https://doi.org/10.1007/s00330-015-4088-2

[23] Yildirim, D., Samanci, C., Ustabasioglu, F.E., Alis, D., Colakoglu, B., Bakir, A., Tutar, O., Kilavuz, A.E. and Kaur, A. (2016) A Dilemma at Gray Scale Thyroid Ultrasound: Microcalcification or Not? Differentiation with Acoustic Radiation Force Impulse Imaging-Virtual Touch Imaging. Medical Ultrasonography, 18, 452 456. https://doi.org/10.11152/mu-872

[24] Yildirim, D., Gurses, B., Gurpinar, B., Ekci, B., Colakoglu, B., Sahin, M. and Kaur, A. (2013) The Value of Ultrasound Elastography-Guided Fine-Needle Aspiration Biopsy of Thyroid Nodules in Reducing Nondiagnostic Results. Journal of Medical Ultrasound, 40, 169-174. https://doi.org/10.1007/s10396-012-0407-2

[25] Tian, W., Hao, S., Gao, B., Jiang, Y., Zhang, X., Zhang, S., Guo, L., Yan, J. and Luo, D. (2016) Comparing the Diagnostic Accuracy of RTE and SWE in Differentiating Malignant Thyroid Nodules from Benign Ones: A Meta-Analysis. Cellular Physiology and Biochemistry, 39, 2451-2463. https://doi.org/10.1159/000452513

[26] Zhang, Y.Z., Xu, T., Gong, H.Y., Li, C.Y., Ye, X.H., Lin, H.J., Shen, M.P., Duan, Y., Yang, T. and Wu, X.H. (2016) Application of High-Resolution Ultrasound, RealTime Elastography, and Contrast-Enhanced Ultrasound in Differentiating Solid Thyroid Nodules. Medicine (Baltimore), 95, e5329. https://doi.org/10.1097/md.0000000000005329

[27] Tian, W., Hao, S., Gao, B., Jiang, Y., Zhang, S., Guo, L. and Luo, D. (2015) Comparison of Diagnostic Accuracy of Real-Time Elastography and Shear Wave Elastography in Differentiation Malignant from Benign Thyroid Nodules. Medicine (Baltimore), 94, e2312. https://doi.org/10.1097/md.0000000000002312

[28] Jin, L., Xu, C., Xie, X., Li, F., Lv, X. and Du, L. (2017) An Algorithm of Image Heterogeneity with Contrast-Enhanced Ultrasound in Differential Diagnosis of Solid Thyroid Nodules. Ultrasound in Medicine \& Biology, 43, 104-110.

[29] Jiang, J., Shang, X., Wang, H., Xu, Y.B., Gao, Y. and Zhou, Q. (2015) Diagnostic Value of Contrast-Enhanced Ultrasound in Thyroid Nodules with Calcification. Kaohsiung Journal of Medical Sciences, 31, 138-144. https://doi.org/10.1016/j.kjms.2014.12.001

[30] Azizi, G., Keller, J.M., Mayo, M.L., Piper, K., Puett, D., Earp, K.M. and Malchoff, C.D. (2015) Thyroid Nodules and Shear Wave Elastography: A New Tool in Thyroid Cancer Detection. Ultrasound in Medicine \& Biology, 41, 2855-2865. https://doi.org/10.1016/j.ultrasmedbio.2015.06.021

[31] Aysan, E., Kiran, T., Idiz, U.O., Guler, B., Akbulut, H., Kunduz, E., Arici, S., Kadakal, G., Ozgor, M. and Coci, K. (2017) The Diagnostic Ability of Core Needle Biopsy in Nodular Thyroid Disease. Annals of the Royal College of Surgeons of England, 99, 233-236.

[32] Eun, N.L., Yoo, M.R., Gweon, H.M., Park, A.Y., Kim, J.A., Youk, J.H., Moon, H.J., Chang, H.S. and Son, E.J. (2016) Thyroid Nodules with Nondiagnostic Results on Repeat Fine-Needle Aspiration Biopsy: Which Nodules Should Be Considered for 
Repeat Biopsy or Surgery Rather than Follow-Up? Ultrasonography, 35, 234-243. https://doi.org/10.14366/usg.15079

[33] Jiang, X.S., Harrison, G.P. and Datto, M.B. (2016) Young Investigator Challenge: Molecular Testing in Noninvasive Follicular Thyroid Neoplasm with Papillary-Like Nuclear Features. Cancer Cytopathology, 124, 893-900. https://doi.org/10.1002/cncy.21802

[34] Pankratz, D.G., Hu, Z., Kim, S.Y., Monroe, R.J., Wong, M.G., Traweek, S.T., Kloos, R.T., Walsh, P.S. and Kennedy, G.C. (2016) Analytical Performance of a Gene Expression Classifier for Medullary Thyroid Carcinoma. Thyroid, 26, 1573-1580. https://doi.org/10.1089/thy.2016.0262

[35] Mohamed, H.E., Mohamed, S.E., Anwar, M.A., Al-Qurayshi, Z., Sholl, A., Thethi, T., Khan, A., Aslam, R. and Kandil, E. (2016) The Significance of Enlarged Cervical Lymph Nodes in Diagnosing Thyroid Cancer. Journal of Cancer Research and Therapeutics, 12, 1006-1009.

[36] Kannan, S., Chauhan, S., Naveen, Latha, B.S., Raju, N., Chandrasekhar, N.H., Kekatpure, V., Kuriakose, M.A. and Manjunath, P. (2016) Estimation of Thyroglobulin in Lymph Node Aspirates: Pilot Experience from a Tertiary Referral Cancer Center. Indian Journal of Endocrinology and Metabolism, 20, 359-363. https://doi.org/10.4103/2230-8210.179987

[37] Mao, F., Xu, H.X., Zhao, C.K., Bo, X.W., Li, X.L., Li, D.D., Liu, B.J., Zhang, Y.F., Xu, J.M. and Qu, S. (2017) Thyroid Imaging Reporting and Data System in Assessment of Cytological Bethesda Category III Thyroid Nodules. Clinical Hemorheology and Microcirculation, 65, 163-173.

[38] Lee, Y.B., Cho, Y.Y., Jang, J.Y., Kim, T.H., Jang, H.W., Chung, J.H., Oh, Y.L. and Kim, S.W. (2017) Current Status and Diagnostic Values of the Bethesda System for Reporting Thyroid Cytopathology in a Papillary Thyroid Carcinoma-Prevalent Area. Head \& Neck, 39, 169-274.

[39] Leboulleux, S., Tuttle, R.M., Pacini, F. and Schlumberger, M. (2016) Papillary Thyroid Microcarcinoma: Time to Shift from Surgery to Active Surveillance? The Lancet Diabetes \& Endocrinology, 4, 933-942. https://doi.org/10.1016/S2213-8587(16)30180-2

[40] Gao, X., Zhang, X., Zhang, Y., Hua, W., Maimaiti, Y. and Gao, Z. (2016) Is Papillary Thyroid Microcarcinoma an Indolent Tumor?: A Retrospective Study on 280 Cases Treated with Radioiodine. Medicine (Baltimore), 95, e5067. https://doi.org/10.1097/md.0000000000005067

[41] Bhatia, K.S., Lam, A.C., Pang, S.W., Wang, D. and Ahuja, A.T. (2016) Feasibility Study of Texture Analysis Using Ultrasound Shear Wave Elastography to Predict Malignancy in Thyroid Nodules. Ultrasound in Medicine \& Biology, 42, 1671-1680. https://doi.org/10.1016/j.ultrasmedbio.2016.01.013

[42] Sahu, N. and Padhy, R.N. (2016) Bayesian Analysis of High-Resolution Ultrasonography and Guided Fine Needle Aspiration Cytology in Diagnosis of Palpable Thyroid Nodules. Brazilian Journal of Otorhinolaryngology. https://doi.org/10.1016/j.bjorl.2016.10.010

[43] Khaled, H., Al Lahloubi, N. and Rashad, N. (2016) A Review on Thyroid Cancer during Pregnancy: Multitasking Is Required. Journal of Advanced Research, 7, 565 570. https://doi.org/10.1016/j.jare.2016.02.007

[44] Bauer, A.J. and Francis, G.L. (2016) Evaluation and Management of Thyroid Nodules in Children. Current Opinion in Pediatrics, 28, 536-544. https://doi.org/10.1097/MOP.0000000000000364

[45] Parisi, M.T., Eslamy, H. and Mankoff, D. (2016) Management of Differentiated 
Thyroid Cancer in Children: Focus on the American Thyroid Association Pediatric Guidelines. Seminars in Nuclear Medicine, 46, 147-164.

https://doi.org/10.1053/j.semnuclmed.2015.10.006

Submit or recommend next manuscript to SCIRP and we will provide best service for you:

Accepting pre-submission inquiries through Email, Facebook, LinkedIn, Twitter, etc. A wide selection of journals (inclusive of 9 subjects, more than 200 journals)

Providing 24-hour high-quality service

User-friendly online submission system

Fair and swift peer-review system

Efficient typesetting and proofreading procedure

Display of the result of downloads and visits, as well as the number of cited articles

Maximum dissemination of your research work

Submit your manuscript at: http://papersubmission.scirp.org/

Or contact jct@scirp.org 